\title{
Efecto del cadmio sobre la germinación y crecimiento de los mangles: Rhizophora mangle, Hilairanthus germinans y Conocarpus erectus
}

\section{Cadmium effect on germination and growth of mangroves: Rhizophora mangle, Avicennia germinans and Conocarpus erectus}

\section{Nelson José Villavicencio Bedor ${ }^{1 \star}$ \& Beatriz Pernía Santos²}

\author{
'Egresado Facultad de Ciencias Naturales, Universidad de Guayaquil \\ ${ }^{2}$ Docente-investigadora, Universidad de Guayaquil, Facultad de Ciencias Naturales
}

Recibido de agosto 2020; recibido en forma revisada 26 de agosto 2020, aceptado 3 de octubre 2020 Disponible en línea 20 de diciembre 2020

\begin{abstract}
Resumen
Los manglares pueden ser afectados por contaminantes que el ser humano genera al ser liberados en las aguas residuales, éstos se transportan a través de los ríos, siendo los metales pesados los que han recibido una mayor atención debido a que poseen una alta toxicidad y no son biodegradables. Entre los metales pesados contaminantes que afectan a los ecosistemas marinos costeros tenemos al cadmio, este es considerado como uno de los más dañinos debido al grado de toxicidad, solubilidad y capacidad de concentrarse e incorporarse en la red trófica. Con lo anteriormente mencionado, es de suma importancia determinar el efecto que posee el $\mathrm{Cd}$ en la germinación y crecimiento en diferentes especies de mangles, como: Rhizophora mangle, Hilairanthus germinans y Conocarpus erectus. Se obtuvo un total de 1.176 hipocótilos y propágulos, éstos fueron esterilizados y sometidos a concentraciones realistas de $\mathrm{Cd}$ de $0.25,0.50,1,2,4$ y 8 ppm, usando agua destilada como testigo positivo. Los estímulos de crecimiento en longitud del hipocótilo no presentaron diferencias significativas entre las concentraciones estudiadas de Cd. La longitud de la radícula en concentraciones mayores de Cd presentó valores nulos de crecimiento. Los valores de porcentaje de germinación en las distintas concentraciones de $\mathrm{Cd}$ en las especies estudiadas se presentaron significativamente menores al testigo. Finalmente, el IIF demostró que en todas las concentraciones de Cd se presentó toxicidad. Se puede concluir que el Cd es tóxico para Rhizophora mangle, Hilairanthus germinans y Conocarpus erectus en niveles permitidos en la normativa ambiental ecuatoriana vigente, por lo que se recomienda reducir el límite máximo permisible de toxicidad de $\mathrm{Cd}$ en Ecuador.
\end{abstract}

Palabras claves: cadmio, germinación, hipocótilo, manglares, radícula.

\begin{abstract}
Mangrove ecosystems can be affected by pollutants that humans generate when released into wastewater and that are downstream transported by rivers, among those, heavy metals have received the most attention because they have a high toxicity and are not biodegradable. Cadmium is a polluting heavy metal that may affect coastal marine ecosystems, that is considered one of the most harmful due to the high degree of toxicity, solubility and ability to concentrate and incorporate into the food web. Consequently, it is of high importance to determine the effect that $\mathrm{Cd}$ has on germination and growth in different species of mangrove trees as Rhizophora mangle, Hilairanthus germinans and Conocarpus erectus. A total of 1,176 hypocotyls and propagules were obtained, those were sterilized and submitted to realistic Cd concentrations of $0.25,0.50,1,2,4$ and $8 \mathrm{ppm}$, using distilled water as a positive control. The growth stimuli of hypocotyl length did not present significant differences between the concentrations studied of $\mathrm{Cd}$. The length of the radicle in higher concentrations of $\mathrm{Cd}$ presented null values in terms of growth. The germination percentage values in the different $\mathrm{Cd}$ concentrations in the studied species were significantly lower than the control. Finally, the Fll showed that toxicity occurred in all concentrations of $\mathrm{Cd}$ and that it can be concluded that $\mathrm{Cd}$ is toxic to Rhizophora mangle, Hilairanthus germinans and Conocarpus erectus at levels permitted in the regulations. Therefore, it is recommended to reduce the maximum permissible limit of $\mathrm{Cd}$ toxicity in Ecuador.
\end{abstract}

Keywords: cadmium, germination, hypocotyl, mangroves, radicle.

* Correspondencia del autor:

E-mail: nelson.villavicenciob@ug.edu.ec 


\section{Introducción}

Los manglares son ecosistemas que se encuentran en el perfil costero, están ubicados dentro del cinturón tropical, usualmente se localizan en las desembocaduras de los ríos hacia el mar, contribuyen a la productividad primaria y son considerados zonas de refugio, desove y alimentación de docenas de especies bioacuáticas (Ulanie-Rosas et al., 2018; Cornejo, 2014). Se encuentran constituidos por formaciones vegetales dominadas por árboles y arbustos conocidos como mangles, éstos poseen características morfológicas y fisiológicas especializadas que les permiten subsistir exitosamente en estos ambientes. Los mangles se distribuyen en una zona de transición entre el ecosistema terrestre y marino, proporcionando hábitat, protección y alimento para muchas poblaciones de animales residentes y migratorios, favoreciendo su desarrollo y subsistencia (Ulanie-Rosas et al., 2018; Gómez-Pais, 2005). Las funciones que poseen los mangles junto con su entorno permiten que el manglar sea un hábitat de alta productividad que aporta con los nutrientes necesarios, estos son la base de una cadena trófica que sustenta una abundante fauna de alto valor ecológico, nutricional y económico (Flores, 2016). Por esta razón, el ecosistema manglar desempeña un rol importante en el desarrollo y subsistencia de las poblaciones humanas en las zonas marino-costeras desde los inicios de los primeros asentamientos humanos en la costa de Ecuador, hace más de 10.000 años AC, siendo actualmente una gran fuente de productividad para la pesca artesanal de cangrejos, conchas y peces (Stothert, 2011; Gómez-Pais, 2005).

Los manglares pueden ser afectados por contaminantes que el ser humano genera al ser liberados en las aguas residuales y que se transportan a éstos a través de los ríos, siendo los metales pesados los que han recibido mayor atención debido a que poseen una alta toxicidad y no son biodegradables, éstos llegan a ser tóxicos, incluso hasta en bajas concentraciones, llegándose a acumular en organismos bioacuáticos en los que se produce un proceso de bioacumulación y posterior biomagnificación (Pernía et al., 2008, 2018).

En la actualidad los manglares sufren alteraciones debido a la contaminación generada por las descargas de aguas servidas e industriales (Foroughbakhch et al., 2004; Vannucci, 2001). Contrario a diversos contaminantes orgánicos, los metales pesados entre ellos: $\mathrm{Sb}, \mathrm{As}, \mathrm{Cd}, \mathrm{Cu}, \mathrm{Cr}, \mathrm{Hg}, \mathrm{Ni}, \mathrm{Pb}, \mathrm{Se}, \mathrm{y} \mathrm{Zn}$, generalmente no se eliminan de los ecosistemas acuáticos por procesos naturales debido a que no son biodegradables, son tóxicos en bajas concentraciones y tienden a acumularse en las zonas costeras y estuarinas (Ramírez, 2016; Lacerda et al., 1988), en donde los mangles desempeñan un papel de filtro en el proceso de depuración de las aguas, reteniendo en su rizósfera compuestos orgánicos y metálicos, convirtiéndose en un reservorio de metales pesados. No obstante, entre los metales pesados contaminantes que afectan a los ecosistemas marinos costeros tenemos al cadmio (Cd), este es considerado como uno de los más dañinos debido al grado de toxicidad, solubilidad y capacidad de concentrarse e incorporarse en la red trófica, principalmente en las plantas acuáticas (Benavides et al., 2018); además, en peces, aves y mamíferos, genera reducción de crecimiento, anemia, hipertensión disrupciones endócrinas, y en anfibios decrecimiento de la metamorfosis (Benavides et al., 2018; Burger, 2008; Vásquez et al., 2005; Revis et al., 1981; Richardson et al., 1974).

En Ecuador existe contaminación producida por las concentraciones de $\mathrm{Cd}$ en sedimentos de varias zonas manglícolas tales como: El Estero Salado, que posee de $0.50-12.00 \mathrm{mg} / \mathrm{Kg}$ Cd; La Reserva Ecológica Manglares Churute, donde se ha registrado 5,99$15,97 \mathrm{mg} / \mathrm{kg}$ Cd (Proaño \& Pozo, 2016), Estero el Macho, que tiene concentraciones de $0,95 \mathrm{mg} / \mathrm{kg}$ Cd (Ramírez, 2017); Puerto El Morro, con 2,99-4,99 $\mathrm{mg} / \mathrm{kg}$ Cd (Mero, 2010); e Isla Santay, en donde se ha registrado $1,0 \mathrm{mg} / \mathrm{kg}$ Cd (Pernía et al., 2018; Mero et al., 2019). En Ecuador se han registrado 6 especies de mangles, los mangles mayores: Rhizophora mangle, $R$. racemosa (éstos producen al híbrido $R$. x harrisonii), Hilairanthus germinans, Laguncularia racemosa; y, los mangles menores: Pelliceria rhizophorae y Tabebuia palustris, los mismos que están siendo afectados por la contaminación por $\mathrm{Cd}$ en los sedimentos de estas zonas manglícolas (Benavides et al., 2018; Cornejo, 2014). Este metal pesado puede llegar a la planta mediante la raíz y ser albergado en cualquier parte de la misma, produciendo un estrés oxidativo elevando o disminuyendo las moléculas antioxidantes de la planta (Idrees et al., 2015).

Según Ramírez (2002), el Cd reúne cuatro de las características más temidas de un tóxico: 1. Efectos adversos para el hombre y el medio ambiente; 2 . Bioacumulación; 3. Persistencia en el medio ambiente; 4. Viaja grandes distancias con el viento y a través de los cursos de agua. Así mismo, el Cd se puede acumular en el sedimento, donde se convierte en un elemento móvil y podría transferirse a las plantas, en éstas las altas concentraciones podrían generar inhibición del crecimiento y en el desarrollo de las raíces, clorosis y estrés oxidativo (Pernía et al., 2008). Con este antecedente, es indispensable estudiar el efecto de este metal pesado sobre los mangles y especialmente en aquellas especies nativas de la costa de Ecuador.

Con lo anteriormente mencionado, es de importancia determinar el efecto que posee el Cadmio en la germinación y crecimiento de las diferentes especies de mangles en Ecuador. Además, se busca proponer un nuevo límite máximo permisible para el Cadmio dentro de la legislación ecuatoriana actual que permita generar una alternativa metodológica para el manejo eficiente y saludable de estos ecosistemas.

\section{Materiales y Métodos}

\section{Fase de Campo}

\section{Obtención y Recolección de muestras}

Se estableció un total de seis puntos de muestreo, 


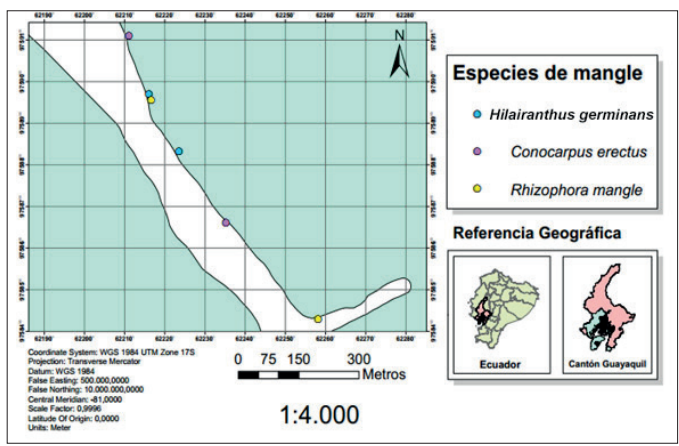

Figura 1. Puntos de muestreo.

fijándose tres puntos de muestreo por cada estación. Se obtuvo un total de 1.176 hipocótilos y semillas, distribuyéndose de la siguiente manera: Rhizophora mangle (336), Hilairanthus germinans (280) y Conocarpus erectus (560). Cabe indicar que las estaciones fueron escogidas debido a que facilitaron el acceso para la toma y el transporte de las muestras (Fig. 1).

Las muestras se colectaron manualmente utilizando guantes de examinación de nitrilo y fueron conservadas en fundas tipo ziploc con cierre hermético. Posteriormente, todas las muestras fueron rotuladas, guardadas y transportadas hasta el Laboratorio de Espectrofotometría del Instituto de Investigaciones de Recursos Naturales (IIRN). Las identificaciones taxonómicas se realizaron en el herbario GUAY de la Facultad de Ciencias Naturales de la Universidad de Guayaquil.

Fase de Laboratorio

Tratamiento de muestras y Procesamiento en laboratorio

Siguiendo la metodología recomendada por Moreno et al. (2013): Se seleccionaron los propágulos para luego lavarlos con agua destilada para remover hongos, tierra o cualquier residuo presente en su superficie. Posteriormente, se retiró manualmente la semilla para ser secadas en toallas de papel absorbente a temperatura ambiente.

Para la determinación del efecto del Cadmio sobre Rhizophora mangle, Hilairanthus germinans, y Conocarpus erectus se utilizó el protocolo propuesto por la Agencia de Protección Ambiental de Estados Unidos (EPA, por sus siglas en inglés) 600/3-88/029 (Greene, 1988): la cual especifica que una vez listas las semillas, estas deben de esterilizarse remojándolas con $\mathrm{NaClO}$ por 30 minutos y posteriormente se lavaron con agua destilada 6 veces, para después ser embebidas por $24 \mathrm{~h}$ en distintas soluciones de cadmio.

Luego, se trabajó con concentraciones realistas de Cd reportadas en suelo ecuatoriano de $0.25,0.50,1$, 2,4 y $8 \mathrm{mg} / \mathrm{L}$, usando agua destilada como testigo positivo. Cabe recalcar, que se realizó un diseño experimental al azar con cuatro réplicas para cada concentración (Pernía et al., 2018).

Para Rhizophora mangle se siguió la metodología usada por Pernía et al. (2018):
Una vez embebidas las muestras en un recipiente de $25 \mathrm{~cm}$ ancho $\times 25 \mathrm{~cm}$ de largo y $30 \mathrm{~cm}$ de altura, se colocaron 10 propágulos por envase junto con tres litros de agua destilada con su respectiva concentración de cadmio $(0.25,0.50,1,2,4$ y 8 $\mathrm{mg} / \mathrm{L})$, también se realizó mediciones de luces y $\mathrm{pH}$ durante 30 días, una vez transcurrido el tiempo de germinación, se midieron y pesaron los hipocótilos y las hojas obtenidas en el proceso, luego se llevó a una estufa a $30^{\circ} \mathrm{C}$ por $24 \mathrm{~h}$, transcurrido ese tiempo se volvió a pesar para obtener peso seco.

En cuanto a Conocarpus erectus y Hilairanthus germinans se trabajó usando la metodología propuesta por Pernía et al. (2018):

Una vez embebidas las semillas por cuadriplicado se colocaron 10 semillas por envase de plástico de 25 $\mathrm{cm}$ ancho diámetro y $10 \mathrm{~cm}$ de altura con tapa a la cual se le realizaron 10 perforaciones para permitir la entrada de aire. A los envases se les colocó una capa de papel filtro Whatman $\mathrm{N}^{\circ} 1$, añadiéndole $5 \mathrm{~mL}$ de la concentración respectiva de $\mathrm{CdNO}_{3}$ (Accustandard).

Los envases con las semillas fueron puestos en una cámara de germinación a $24 \pm 1^{\circ} \mathrm{C}$, humedad $(60-80$ \%), 2000 luz y un fotoperíodo de 12 horas por 30 días.

La longitud de las radículas e hipocótilos se midieron con un vernier calibrado (Stainless) a interdiario a partir de la primera germinación. Simultáneamente se llevó un control de la humedad y la temperatura $\left(25^{\circ} \pm 1^{\circ} \mathrm{C}\right)$ con un termohigómetro y la luminosidad con un luxómetro (Kyoritsu).

Las concentraciones inhibitorias medias (Cl50) para los bioensayos se determinaron utilizando el Programa Probit de la Agencia de Protección Ambiental de los Estados Unidos (USEPA), empleando el método de interpolación lineal para toxicidad subletal y calculando la concentración de inhibición media de la longitud de la radícula (Pernía et al., 2018).

\section{Procesamiento de Datos}

El porcentaje de germinación se determinó a diario según la fórmula de (Cokkizgin y Cokkizgin, 2010) (Cuadro 1), así mismo se calculó el tiempo medio de germinación (TMG) y la velocidad media de germinación (VMG) (Moreno et al., 2013).

Tabla 1. Fórmulas a utilizar en el cálculo de las variables de estudio.

\begin{tabular}{lll}
\hline \multicolumn{1}{c}{ Variable } & \multicolumn{1}{c}{ Fórmula } & \multicolumn{1}{c}{ Autor } \\
\hline Porcentaje de & PG = número de & Cokkizgin y \\
germinación $(P G)$ & $\begin{array}{l}\text { semillas germinadas } \\
\text { x100/número de }\end{array}$ & Cokkizgin, \\
& se10
\end{tabular}
semillas totales

$\begin{array}{lll}\begin{array}{l}\text { Tiempo medio } \\ \text { de germinación } \\ \text { (TMG) }\end{array} & \mathrm{TMG}=(\mathrm{x} 1 \mathrm{~d} 1)+\ldots(\mathrm{xn} \mathrm{dn})) / \mathrm{Xn} & \begin{array}{l}\text { Moreno et } \\ \text { al., }\end{array} \\ \begin{array}{l}\text { Velocidad media } \\ \text { de germinación }\end{array} & \mathrm{VMG}=\mathrm{P} 1 / \mathrm{T} 1+\mathrm{P} 2 / & \text { Moreno et } \\ \text { (VMG) } & & \text { al., } \\ \end{array}$


$\mathrm{P}=$ número de semillas germinadas; $\mathrm{T}=$ tiempo germinación; $\mathrm{n}$ = día último control; $\mathrm{x} 1, \mathrm{x} 2, \mathrm{x} 15=$ semillas germinadas día $1,2, \ldots \mathrm{n}$; d1, d2,...dn = días incubación; $\mathbf{X i}=$ semillas germinadas por día de revisión; $\mathrm{Xn}$ = número total de semillas germinadas el último día de control; y Tn = último día del ensayo.

\section{Índice de tolerancia (IT)}

El índice de tolerancia (IT) se determinó mediante la ecuación propuesta por (Wilkins, 1978):

$$
\mathrm{IT}=\frac{L R m}{L R c}
$$

Donde:

$\mathrm{LRm}$ = es la longitud radical de las plántulas que crecieron en presencia del cadmio.

$\mathrm{LRC}=$ es la longitud radical de las plántulas en ausencia del cadmio.

\section{Índice integral de fitotoxicidad (IIF)}

Se determinó la toxicidad del cadmio utilizando el índice integral de fitotoxicidad según la fórmula propuesta por Pernía et al. (2018):

Donde:

$$
I I F=100-\left(\frac{S G M}{S G C}\left(\frac{\frac{L R M}{L R C}+\frac{L H M}{L H C}}{2}\right) 100\right)
$$

SGM = número de semillas germinadas de la muestra, es el promedio del número de semillas germinadas en las cuatro réplicas para cada tratamiento $(n=4)$.

$\mathrm{SGC}=$ número de semillas germinadas del control, es el promedio del número de semillas germinadas en las cuatro réplicas del testigo $(n=4)$.

LRM = Longitud de la radícula de la muestra, es el promedio de la medición en centímetros de las radículas de 10 plántulas por réplica de cada tratamiento $(n=40)$.

LRC = Longitud de la radícula del control, es el promedio de la medición en centímetros de las radículas de 10 plántulas por réplica del testigo $(\mathrm{n}=$ 40).

LHM = Longitud del hipocótilo de la muestra, es el promedio de la medición en centímetros de los hipocótilos de 10 plántulas por réplica de cada tratamiento $(n=40)$.

LHC = Longitud del hipocótilo del control, es el promedio de la medición en centímetros de los hipocótilos de 10 plántulas por réplica del testigo $(n=40)$.

Este índice muestra los resultados de -100 a 100 pudiéndose interpretar como porcentaje, mostrando la inhibición de crecimiento con valores positivos y estímulo de crecimiento con valores negativos.

\section{Análisis estadístico}

Los resultados se muestran como medias \pm desviación estándar. Se analizó la normalidad de los datos utilizando la prueba de Anderson-Darling y la igualdad de varianza con el test de Levene. Para determinar si existían diferencias significativas en el porcentaje de germinación expuestas a las diferentes concentraciones de Cd se aplicó una prueba de ANOVA de una vía y test a Posteriori de Tukey $(P \geq 0.05)$. Para los datos no paramétricos se utilizó la prueba de Kruskall-Wallis. Para todos los análisis se utilizó el programa MINITAB versión 19.

\section{Resultados}

\section{Efectos del Cd sobre la longitud del hipocótilo y la radícula}

Durante el estudio para Rhizophora mangle se trabajó un total de 336 hipocótilos con 6 tratamientos de diferentes concentraciones de $\mathrm{Cd}$ más un blanco (agua destilada), dividiéndose 48 hipocótilos por tratamiento. Se observó mayores estímulos de crecimiento para los tratamientos de 0,25 ppm, 0,50 ppm y 1 ppm presentando una longitud de 3,77 $\pm 1,85$ $\mathrm{cm}, 3,58 \pm 1,35 \mathrm{~cm}$ y $3,46 \pm 0,85 \mathrm{~cm}$ respectivamente menores al testigo $(4,18 \mathrm{~cm})$. Aunque, se evidenció que el tratamiento de $2 \mathrm{ppm}$ con una longitud de 2,37 $\pm 1,03 \mathrm{~cm}$ presentó un menor estímulo de crecimiento que los mencionados anteriormente.

Por el contrario, la longitud de la radícula mostró un valor de 3,17 $\pm 1,11 \mathrm{~cm}$ para el tratamiento de 0,25 ppm menor a la longitud del blanco $(3,72 \mathrm{~cm})$, así como los valores $1,64 \pm 0,67 \mathrm{~cm}$ para $0,50 \mathrm{ppm}$ y 0,57 $\pm 0 \mathrm{~cm}$ para $1 \mathrm{ppm}$, respectivamente. Sin embargo, en los tratamientos de $2 \mathrm{ppm}, 4 \mathrm{ppm}$ y $8 \mathrm{ppm}$ se obtuvieron valores nulos en cuanto a longitud.

Para Conocarpus erectus se utilizó un total de 560 semillas con 6 tratamientos de diferentes concentraciones de $\mathrm{Cd}$ más un blanco (agua destilada), dividiéndose 20 semillas por tratamiento, observándose un valor $0,42 \pm 0,30 \mathrm{~cm}$ para el tratamiento de 0,25 ppm mayor a la longitud del blanco $(0,22 \mathrm{~cm})$, así mismo se observó una longitud del hipocótilo de $0,40 \pm 0,47 \mathrm{~cm}$ para el tratamiento de $1 \mathrm{ppm}$ también mayor al valor mostrado por el testigo, en cambio se observó una longitud del hipocótilo de

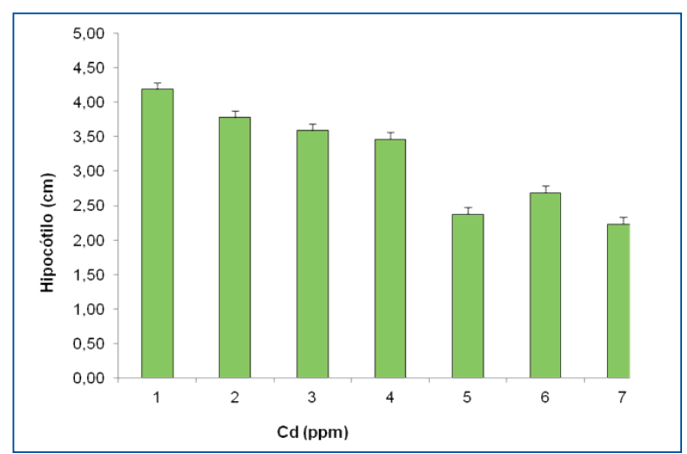

Figura 2. Longitud de hipocótilo de plántulas de Rhizophora mangle en distintas concentraciones de Cadmio (ppm). Los resultados obtenidos se presentan como barras representando la Media \pm DS. 


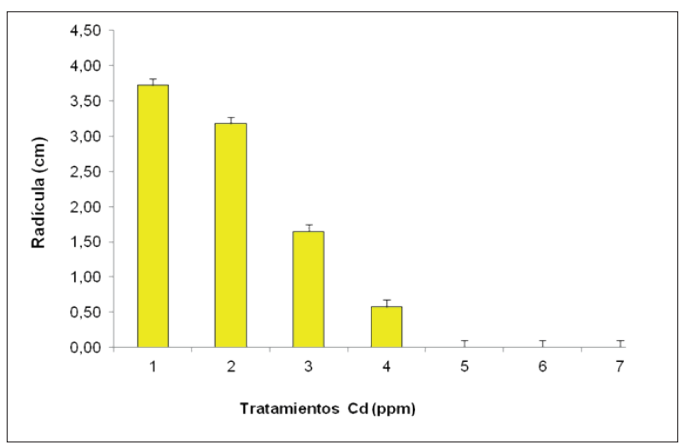

Figura 3. Longitud de las radículas de plántulas de Rhizophora mangle en distintas concentraciones de Cadmio (ppm). Los resultados obtenidos se presentan como barras representando la Media \pm DS.

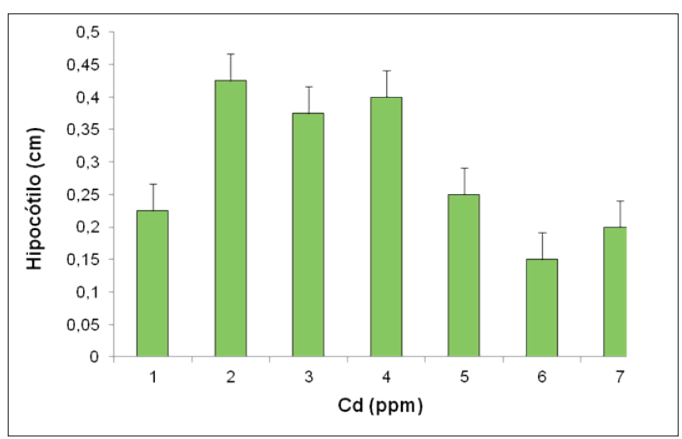

Figura 4. Longitud de hipocótilo de plántulas de Conocarpus erectus en distintas concentraciones de Cadmio ( $\mathrm{ppm})$. Los resultados obtenidos se presentan como barras representando la Media \pm DS.

$0,15 \pm 0,17 \mathrm{~cm}$ para el tratamiento de $4 \mathrm{ppm}$ menor a la longitud del blanco $(0,22 \mathrm{~cm})$.

En cuanto a la longitud radicular se detectó un valor en longitud de $1,75 \pm 1,38 \mathrm{~cm}$ para el tratamiento de $0,25 \mathrm{ppm}(1)$ menor a la longitud del testigo $(2 \mathrm{~cm}) \mathrm{y}$ un valor $0,40 \pm 0,30 \mathrm{~cm}$ para el tratamiento de $8 \mathrm{ppm}$ (6) que también es menor a la longitud del blanco. Además, se observó necrosis radicular en las muestras tratadas con 2, 4 y 8 ppm de concentración de $\mathrm{Cd}$, respectivamente.

En Hilairanthus germinans se trabajó un total de 280 semillas con 6 tratamientos de diferentes concentraciones de Cd más un blanco (agua destilada), dividiéndose 40 semillas por tratamiento. Se evidenció mayor estímulo de crecimiento para el tratamientos de 0,25 ppm presentando una longitud del hipocótilo de 0,07 $\pm 0,17 \mathrm{~cm}$ menor al testigo $(0,11 \mathrm{~cm})$. Así mismo, se observó que los tratamientos con 0,50, 1 y 2 ppm respectivamente el estímulo de crecimiento se mantiene constante y a su vez menores al blanco $(0,11$ $\mathrm{cm})$.

Por otra parte, se pudo observar para la longitud de la radícula un valor $1,00 \pm 3,66 \mathrm{~cm}$ para el tratamiento de $0,25 \mathrm{ppm}$ igual al obtenido por el control $(1,00$

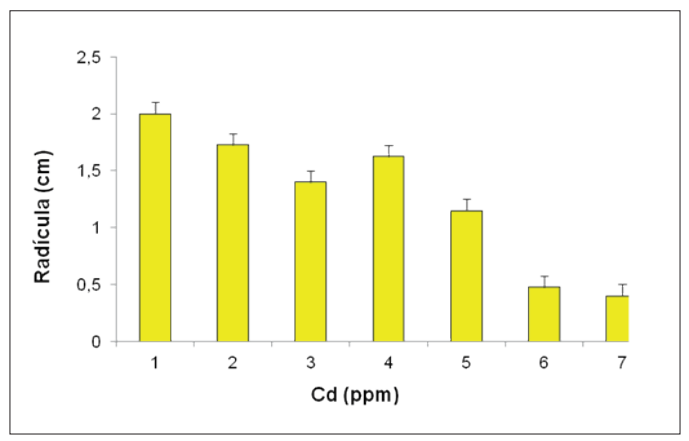

Figura 5. Longitud de las radículas de plántulas de Conocarpus erectus en distintas concentraciones de Cadmio (ppm). Los resultados obtenidos se presentan como barras representando la Media \pm DS.

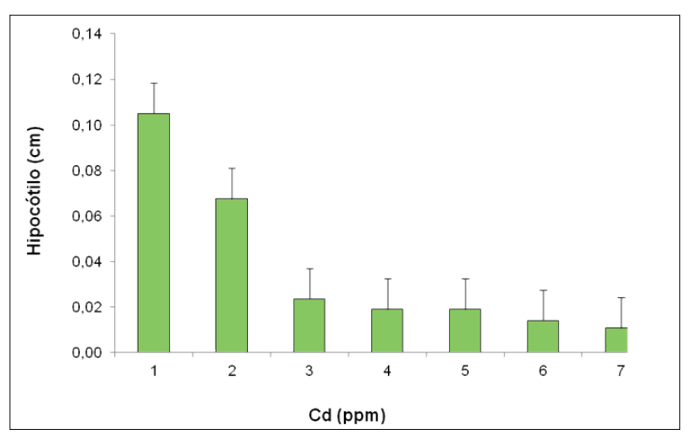

Figura 6. Longitud de hipocótilo de plántulas de Hilairanthus germinans en distintas concentraciones de Cadmio (ppm). Los resultados obtenidos se presentan como barras representando la Media \pm DS

$\mathrm{cm})$. No obstante, en los tratamientos siguientes se obtuvieron valores de longitud de la radícula menores al testigo $(1,00 \mathrm{~cm})$. Además, se observó una coloración marrón (necrosis radicular) en las raíces expuestas a concentraciones de 2, 4 y 8 ppm de $\mathrm{Cd}$, respectivamente.

\section{Efectos del Cd sobre la germinación de Rhizophora mangle, Hilairanthus germinans y Conocarpus erectus}

En Rhizophora mangle se muestra que el mayor porcentaje de germinación se registró a 0,25 ppm de concentración de $\mathrm{Cd}(57 \% \pm 0,25 \%)$, sin embargo, es menor al obtenido en el control $(74 \% \pm 0,28 \%)$. El menor porcentaje de germinación fue de $11 \% \pm 0,27 \%$ a 2, 4 y 8 ppm de concentración de $C d$ respectivamente, resultando en secuencia y significativamente menores al testigo $(74 \% \pm 0,28 \%)$.

Por el contrario, se evidenció que el mayor porcentaje de germinación en Conocarpus erectus se registró para el control $(28,16 \% \pm 3,49 \%)$, seguido por $0,25 \mathrm{ppm}$ de concentración de $\mathrm{Cd}(25,83 \% \pm 3,47 \%)$. No obstante, el menor porcentaje de germinación se observó para las concentraciones de 4 y 8 ppm respectivamente y en secuencia $(7,33 \% \pm 3,50 \%)$. 


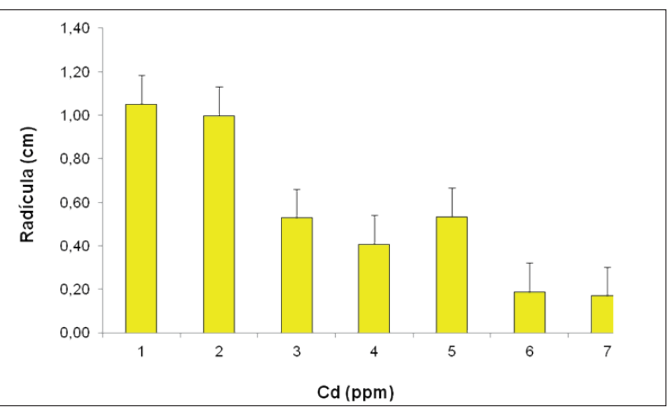

Figura 7. Longitud de las radículas de plántulas de Hilairanthus germinans en distintas concentraciones de Cadmio ( $\mathrm{ppm})$. Los resultados obtenidos se presentan como barras representando la Media \pm DS.

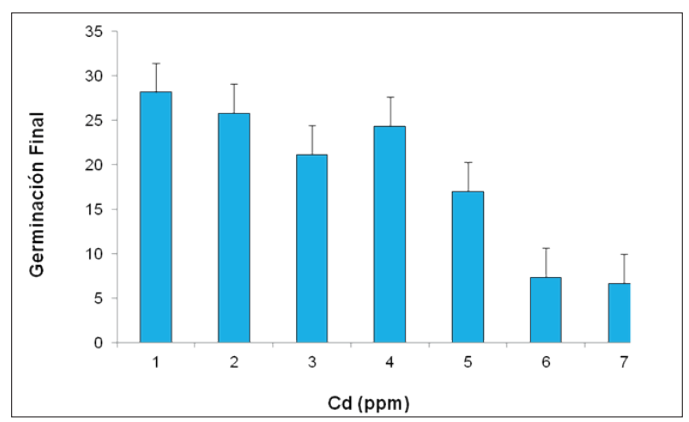

Figura 9. Porcentaje de germinación final de Conocarpus erectus expuesta a diferentes concentraciones de $\mathrm{Cd}(0,0,25$, $0,50,1,2,4,8 \mathrm{ppm})$. Los resultados obtenidos se presentan como barras representando la Media \pm DS.

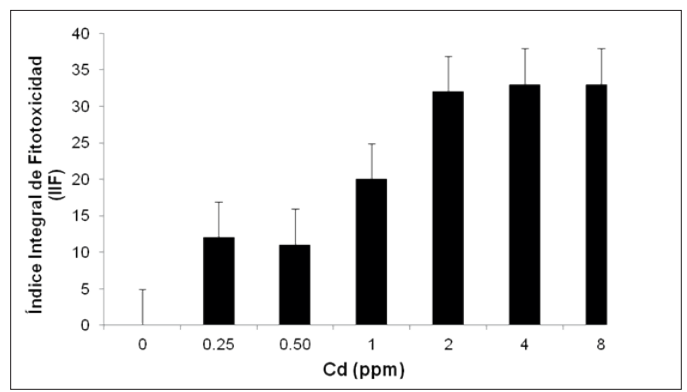

Figura 11. Índice Integral de fitotoxicidad de las plántulas de Rhizophora mangle expuestas a diferentes concentraciones de $\mathrm{Cd}(0,0,25,0,50,1,2,4,8 \mathrm{ppm})$. Los resultados obtenidos se presentan como barras representando la Media \pm DS.

Para Hilairanthus germinans se muestra que el mayor porcentaje en germinación se registró en valores similares a $0,25 \mathrm{ppm}$ de concentración de $C d(100 \% \pm 31,50)$ y al control $(100 \% \pm 31,39$ $\%)$, y a 2 ppm de concentración de Cd $(72,56 \% \pm$ $31,40)$. El menor porcentaje de germinación fue de $25,93 \% \pm 31,49$ a $4 \mathrm{ppm}$ de concentración de Cd y de $22,71 \% \pm 32,30$ a 8 ppm de concentración de $\mathrm{Cd}$, respectivamente, siendo significativamente menores al control.

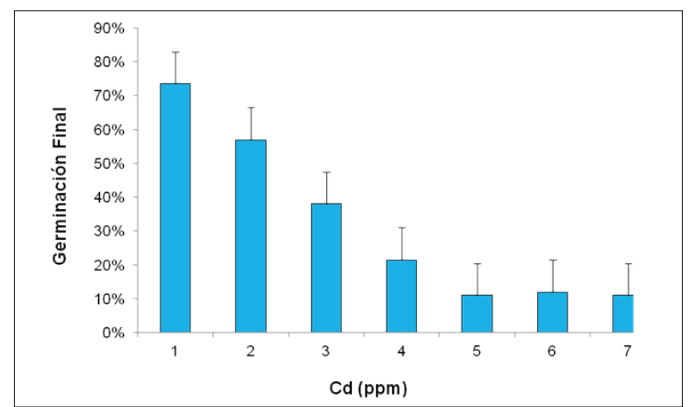

Figura 8. Porcentaje de germinación final de Rhizophora mangle expuesta a diferentes concentraciones de $\mathrm{Cd}(0,0,25$, $0,50,1,2,4,8 \mathrm{ppm})$. Los resultados obtenidos se presentan como barras representando la Media \pm DS.

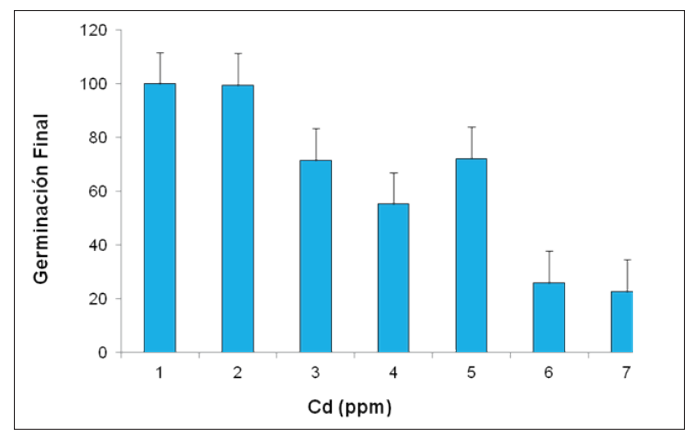

Figura 10. Porcentaje de germinación final de Hilairanthus germinans expuesta a diferentes concentraciones de $\mathrm{Cd}$ $(0,0,25,0,50,1,2,4,8 \mathrm{ppm})$. Los resultados obtenidos se presentan como barras representando la Media \pm DS.

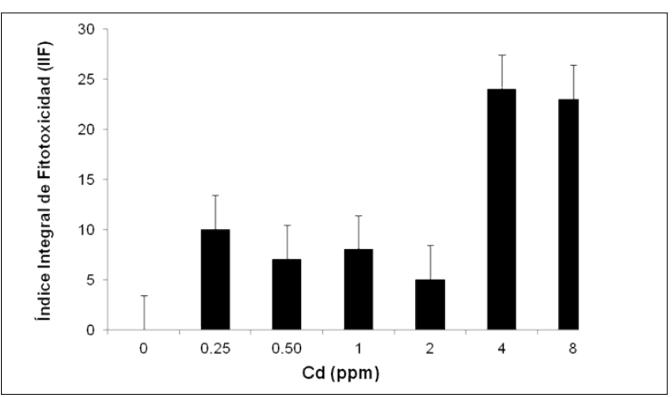

Figura 12. Índice Integral de fitotoxicidad de las plántulas de Conocarpus erectus expuestas a diferentes concentraciones de Cd (0, 0,25, 0,50, 1, 2, 4, 8 ppm). Los resultados obtenidos se presentan como barras representando la Media \pm DS.

\section{Índice Integral de fitotoxicidad (Cd)}

El Índice Integral de Fitotoxicidad (IIF) manifiesta la visión integral de la toxicidad del Cd, en Rhizophora mangle se aprecia que en todas las concentraciones de este metal pesado se presentó toxicidad, siendo los valores resultantes más altos para las concentraciones de $\mathrm{Cd}$ de 2, 4, 8 ppm con 33,1, respectivamente.

Del mismo modo, se obtuvo el Índice Integral de Fitotoxicidad (IIF) para Conocarpus erectus, se 


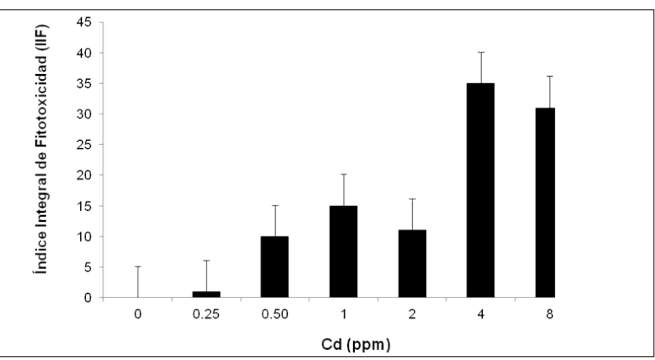

Figura 13. Índice Integral de fitotoxicidad de las plántulas de Hilairanthus germinans expuestas a diferentes concentraciones de $\mathrm{Cd}(0,0,25,0,50,1,2,4,8 \mathrm{ppm})$. Los resultados obtenidos se presentan como barras representando la Media \pm DS

pudo observar que todas las concentraciones de $\mathrm{Cd}$ presentaron toxicidad, y los valores más altos 24,1 y 23,6 se presentaron a 4 y 8 ppm, respectivamente. Así mismo, se obtuvo el Índice Integral de Fitotoxicidad (IIF) para Hilairanthus germinans, se pudo constatar que todas las concentraciones de $\mathrm{Cd}$ presentaron toxicidad, siendo los valores más altos 35,1 y 31,8 para 4 y 8 ppm de $\mathrm{Cd}$, respectivamente.

\section{Discusión}

El cadmio, metal pesado tóxico debido a sus diversas características puede llegar a las plantas mediante la raíz y se alberga en cualquier parte de la misma, produciendo un estrés oxidativo elevando o disminuyendo las moléculas antioxidantes de la planta haciendo indispensable estudiar el efecto de este metal sobre los mangles (Idrees et al., 2015). Por esta razón se trabajó con los hipocótilos y radículas de Rhizophora mangle, Conocarpus erectus y Hilairanthus germinans, en donde se pudo constatar que la longitud del hipocótilo no fue un buen indicador de la toxicidad del $\mathrm{Cd}$, debido a que los valores en longitud del hipocótilo de las plántulas de mangle expuestas a las diferentes concentraciones de $\mathrm{Cd}$ no mostraron diferencias significativas con respecto al testigo en cuanto a concentraciones menores.

Sin embargo, en los tratamientos con mayores concentraciones sí se observó diferencias significativas con respecto al testigo. Aunque, si se compara con los resultados obtenidos en por Liu et al. (2012), quienes observaron que las plantas de Suaeda salsa (Amaranthaceae) no germinaron (inhibición) al exponerlas a concentraciones de 0,1 -6,0 mg/L de $\mathrm{Cd}$, las especies de mangles aquí estudiadas son más resistentes y esto podría deberse a que las muestras fueron obtenidas en zonas con antecedentes de contaminación por Cd (Estero Salado) y las mismas tienen tolerancia a dicho metal (Fernández-Cadena et al., 2014). Además, hay que considerar que se trabajó en condiciones específicas y controladas de laboratorio.

Por otra parte, la longitud de la radícula mostró afectación en cuanto a los datos obtenidos ya que esta se reducía conforme aumentaban las concentraciones de Cd (2, 4, 8 ppm). Así mismo, se observó necrosis radicular (coloración café) en las raíces expuestas a altas concentraciones de $\mathrm{Cd}$, fenómeno observado también por otros autores (Benavides et al., 2018; Vassilev \& Yordanov, 1997), y estos últimos mencionan que los síntomas de fitotoxicidad se expresan más claramente en las raíces debido a que estas acumulan grandes concentraciones del metal pesado. Otros estudios demuestran que otras especies son más sensibles al Cd, tal como el realizado por He et al. (2008), quienes publicaron que existe un efecto marcado sobre el crecimiento de la radícula de plántulas de Oryza sativa (Poaceae) expuesta a 5 - 25 umol / L de Cd.

La germinación de las plántulas de Rhizophora mangle, Conocarpus erectus y Hilairanthus germinans no presentaron diferencias significativas hacia las menores concentraciones de $\mathrm{Cd}$ con respecto al blanco. No obstante, se observó que el porcentaje de germinación de las plántulas resultó menor con respecto al testigo en concentraciones mayores de Cd. Contrario a estos resultados, Benavides et al. (2018), registró a $4 \mathrm{mg} / \mathrm{L}$ de $\mathrm{Cd}(82,50 \pm 17,07$ y a 1 $\mathrm{mg} / \mathrm{L} \mathrm{Cd}(80,00 \% \pm 14,14 \%)$ al trabajar con Laguncularia racemosa var. glabriflora; sin embargo, este resultado podría deberse a que se trata de un género diferente de mangle y se trabajaron en condiciones controladas de laboratorio.

Así mismo, los mismos autores mencionados detallan que la germinación se ha descrito como un parámetro poco sensible a otros contaminantes como hidrocarburos y metil terbutil éter. Además, en el presente trabajo se utilizaron altas concentraciones de $\mathrm{Cd}$ de manera similar que en otros (An, 2004; Pernía et al. 2007), y no se observó inhibición de la germinación al utilizar altas concentraciones de $\mathrm{Cd}$ al igual que otros autores y en otras especies como: Cucumis sativus (Cucurbitaceae), Sorghum bicolor, Triticum aestivum, Zea mays (Poaceae), Helianthus annuus (Asteraceae), Lens culinaris, y Phaseolus vulgaris (Fabaceae; op cit).

En cuanto al índice integral de fitotoxicidad (IIF), el cual nos puede mostrar los efectos del Cd sobre la germinación, el crecimiento de los hipocótilos y las radículas, se pudo obtener que todas las concentraciones de $\mathrm{Cd}$ presentaron toxicidad, más aún en las de mayor concentración (4 y 8 ppm) y a su vez estos resultados son similares a los datos hallados sobre IIF por Benavides et al., (2018), con 27,05 y 31,98 , presentándose en las concentraciones de 0,5 y $8 \mathrm{mg} / \mathrm{L} \mathrm{Cd}$.

Finalmente, se puede concluir que el $\mathrm{Cd}$ es tóxico para Rhizophora mangle, Conocarpus erectus y Hilairanthus germinans a niveles permitidos en la normativa ecuatoriana para la preservación de la vida acuática y silvestre en aguas marinas y de estuario (0.5 ppm en sedimentos) (Texto Unificado de la legislación Ambiental). Además, cabe recalcar que estas especies se encuentran como vulnerables por la 
pérdida de su hábitat y la contaminación de las zonas de manglar en el Ecuador.

\section{Conclusiones}

Los estímulos de crecimiento de longitud de hipocótilo no presentaron diferencias significativas entre las concentraciones estudiadas de Cd para Rhizophora mangle, Hilairanthus germinans, y el control. No así para Conocarpus erectus que presentó valores mayores al blanco.

La longitud de la radícula en concentraciones mayores de Cd (2, 4, 8 ppm) presentó valores nulos, en cambio para Conocarpus erectus se observaron valores menores al control al igual que para Hilairanthus germinans.

En Rhizophora mangle, Hilairanthus germinans, y Conocarpus erectus se evidenció necrosis radicular en las raíces expuestas a concentraciones de 2, 4 y 8 ppm de $\mathrm{Cd}$, respectivamente. Esto probablemente se debe a que los síntomas de fitotoxicidad se expresan más claramente en las raíces.

Los valores de porcentaje de germinación de Rhizophora mangle, Hilairanthus germinans, y Conocarpus erectus en las distintas concentraciones de $\mathrm{Cd}$ se presentaron significativamente menores al testigo.

El Índice Integral de Fitotoxicidad (IIF) manifestó la visión integral de la toxicidad del Cd en Rhizophora mangle, Hilairanthus germinans, y Conocarpus erectus, debido a que se confirmó que en todas las concentraciones del metal pesado se presentó toxicidad y así mismo que los valores más altos se obtuvieron en las concentraciones de 4 y 8 ppm, respectivamente.

\section{Recomendaciones}

- $\quad$ Debido a que el Cd es tóxico para Rhizophora mangle, Hilairanthus germinans, y Conocarpus erectus en niveles permitidos en la normativa ambiental ecuatoriana vigente $(0.5 \mathrm{ppm}$ en sedimentos), es necesario reducir el límite máximo permisible de toxicidad de $\mathrm{Cd}$ en el Texto Unificado de la legislación Ambiental de Ecuador.

- Continuar con la evaluación de cadmio mediante pruebas de laboratorio in situ usando las mismas concentraciones trabajadas.

- Realizar futuros estudios usando las mismas concentraciones trabajadas, pero con otros metales pesados $(\mathrm{Hg}, \mathrm{Cr}, \mathrm{Ni}, \mathrm{Pb}, \mathrm{As}, \mathrm{Zn})$.

- Conocarpus erectus posee una tasa de germinación baja, por lo que se recomienda contrastar las condiciones controladas de laboratorio con condiciones in situ.

- Realizar otros estudios de germinación y crecimiento usando las mismas especies, pero con muestras de distintas zonas marino-costeras.

\section{Bibliografía}

An, Y.-J. 2004. Soil ecotoxicity assessment using cadmium sensitive plants. Environmental Pollution, 127: 21-26. DOI: https: / / doi.org/10.1016/S0269-7491(03)00263-X.

Benavides, A., Del Pezo, R., Pernía, B., Mero, M., Cornejo, X., Magallanes, I., y Romero, B. 2018. Efecto del cadmio sobre la germinación y crecimiento de Laguncularia racemosa var. glabriflora (Plantae-Combretaceae). ECOVIDA, 8: 64-83

Burger, J. 2008. Assessment and Management of Risk to Wildlife from Cadmium. Science of the Total Environment, 389: $37-45$

Cokkizgin, A., y Cokkizgin, H. 2010. Effects of lead $\left(\mathrm{PbCl}_{2}\right)$ stress on germination of lentil (Lens culinaris Medic) lines. African Journal of Biotechnology, 9: 8608-8612. Recuperado https: //doi,org/10,5897/AJB10,890

Cornejo, X. 2014. Plants of the South American Pacific Mangrove swamps (Colombia, Ecuador, Perú), 312 p. Universidad de Guayaquil, Ed. Eduquil.

Fernández-Cadena, J. C., Andrade, S., Silva-Coello, C. L., y De la Iglesia, R. 2014. Heavy metal concentration in mangrove surface sediments from the north-west coast of South America. Marine Pollution Bulletin, 82: 221-226. Disponible en https://doi.org/10.1016/j.marpolbul.2014.03.016

Flores, E. 2016. Criterios metodológicos para la valoración económica de manglares del Ecuador. Tesis de Grado para la obtención de título de Biólogo, Universidad de Guayaquil. Recuperado de http://repositorio,ug,edu,ec/ bitstream / redug / 22235 / 1 / ESTUDIO\%20MANGLAR\%20 REPOSITORIO,pdf

Foroughbakhch, R., Céspedes, A., Alvarado-Vázquez, M., Núñez-González, A. , y Badii, M.H. 2004. Aspectos ecológicos de los manglares y su potencial como fitorremediadores en el Golfo de México. Ciencia UANL, 7: 203-208. Recuperado de http://eprints, uanl, mx/1414/1/art_manglares,pdf

Gómez-Pais, G. 2005. Importancia económico-ambiental del ecosistema manglar. Economía y Desarrollo, 138: 111-134.

Greene, J.C., Bartels, W.J., Warren-Hicks, B.R., Parkhurst, G.L., Linder B.R., Peterson, S.A., y Miller, W.E. 1988. EPA/600/3-88/029, Protocols for short-term toxicity screening of hazardous waste sites, Environmental Research Laboratory, Office of Research and Development, U, S, Environmental Portection Agency, Corvalis, OR, 102 pp.

He, J., Ren, Y., Zhu, C., y Jiang, D. 2008. Effects of Cadmium Stress on Seed Germination, Seedling Growth and Seed Amylase Activities in Rice (Oryza sativa). Rice Science, 15: 319-325. DOI: https://doi.org/10.1016/S16726308(09)60010-X

Idrees, S., Shabir, S., Ilyas, N., Batool, N., y Kanwal, S. 2015. Assessment of cadmium on wheat (Triticum aestivum L.) in hydroponics medium. Agrociencia, 49: 917-929.

Lacerda, L.D., Martinelli, L.A., Rezende, C.E., Mozeto, A., Ovalle, A.R., Victoria, R.L., Silva, C.A., y Nogueira, F.B. 1988. The Fate of Trace Metals in Suspended Matter in a Mangrove Creek during a Tidal Cycle. The Science of the Total Environment, 75: 169-80.

Liu, S., Yang, C., Xie, W., Xia, C., y Fan, P. 2012. The effects of cadmium on germination and seedling growth of Suaeda salsa. Procedia, 16: 293-298. https://doi.org/10.1016/j. proenv.2012.10.041 
Mero, M. 2010. Determinación de metales pesados (Cd y Pb) en moluscos bivalvos de interés comercial de cuatro esteros del Golfo de Guayaquil. Tesis de maestría, Universidad de Guayaquil.

Mero, M., Pernía, B., Ramírez-Prado, N., Bravo, K., Ramírez, L., Larreta, E., y Egas, F. 2019. Concentración de cadmio en agua, sedimentos, Eichhornia crassipes y Pomacea canaliculata en el río Guayas (Ecuador) y sus afluentes. Revista Internacional de Contaminación Ambiental 35: 623-640, DOI: 10,20937/RICA, 2019,35,03,09

Moreno, E., Miranda, D., y Martínez, F. 2013. Zeatin promotes sugar apple seed germination (Annona squamosa L.). Revista Colombiana de Ciencias Hortícolas, 7: 9-19.

Pernía, B., Raimúndez, E., y Castrillo, M. 2007. Resultados preliminares del efecto de la contaminación por cadmio en la germinación de algunas especies de plantas de cultivo en Venezuela. In XVII Congreso Venezolano de Botánica. Maracaibo, Venezuela.

Pernía, B., De Sousa, A., Reyes, R., y Castrillo, M. 2008. Biomarcadores de contaminación por cadmio en plantas. Interciencia, 33: 112-119.

Pernía, B., Rojas-Tortolero, D., Sena, L., De Sisto, A., Inojosa, Y., y Naranjo, L. 2018. Fitotoxicidad de hap, crudos extra pesados y sus fracciones en Lactuca sativa: Una interpretación integral utilizando un índice de toxicidad modificado. Revista Internacional de Contaminación Ambiental, 34: 79-91. Recuperado de https:// doi,org/10,20937/RICA, 2018,34,01,07

Pernía, B., M., Mero, M., Cornejo, X., Ramírez-Prado, N., Ramírez, L., Bravo, K., Zambrano, J. 2018. Determinación de cadmio y plomo en agua, sedimento y organismos bioindicadores en el Estero Salado, Ecuador. Enfoque UTE, 9: 89-105. Recuperado de https://doi,org/10,29019/ enfoqueute, $v 9 \mathrm{n} 2,246$

Proaño, M., y Pozo, F. 2016. Análisis espacial de concentraciones de metales pesados en agua y sedimentos de la reserva ecológica Manglares de Churute, 72. Tesis de maestría, Universidad de Guayaquil. Recuperado de http: / / repositorio,ug,edu,ec/handle/redug/14817

Ramírez, A. 2002. Toxicología del cadmio, Conceptos actuales para evaluar exposición ambiental u ocupacional con indicadores biológicos. Anales de la Facultad de Medicina, Universidad Nacional Mayor de San Marcos, 63: 51-64

Ramírez, N. 2016. Determinación de cadmio en los ríos Guayas, Daule y Babahoyo mediante el estudio de concentraciones en agua, sedimento y en el caracol manzana, Pomacea canaliculata (Lamarck, 1822) (Caenogastropoda: Ampullariidae). Tesis de Grado para la obtención de título de Biólogo, Universidad de Guayaquil.

Ramírez, A. 2017. Determinación de Cadmio y Plomo en agua y sedimento del estero "El Macho" de la ciudad de Machala. Tesis de Grado para la obtención de título de Ingeniero Ambiental, Universidad de Guayaquil. Recuperado de http: / / repositorio,ug,edu,ec/handle/redug/18532

Revis, N.W., Zinsmeister, R., y Bull, R. 1981. Atherosclerosis and Hypertension Induction by Lead and Cadmium Ions: An Effect Prevented by Calcium Ion. Proceedings of the National Academy of Sciences of the United States of America, 78: 6494-6498.

Richardson, M.E., Fox, D., y Fry, B.E. 1974. Pathological Changes Produced in Japanese Quail by Ingestion of Cadmium. The Journal of Nutrition, 104: 323-338.

Stothert, K.E. 2011. Coastal resources and the early Holocene Las Vegas adaptation of Ecuador, In: Trekking the Shore (pp. 355-382), Springer, New York, NY.

Ulanie-Rosas, C., Correa, D., Villegas-Sánchez, C. y CarrilloBastos, A. 2018. Los manglares y su importancia para las pesquerías en Quintana. Revista AvaCient, 5: 89-95.

Vannucci, M. 2001. What is so special about mangroves. Brazilian Journal of Biology, 61: 599-603.

Vassilev, A. y Yordanov, I. 1997. Reductive analysis of factors limiting growth of cadmium-treated plants: a review.
Bulgarian Journal of Plant Physiology, 23: 114-133.

Vásquez, R., Bastardo, A., y Mundarain, I.K. 2005. Ensayo de Toxicidad Aguda CL50-96h con acetato de cadmio y parámetros hematológicos en el híbrido cultivado Colossoma macropomum X Piaractus brachypomus. Zootecnia Tropical, 23: 247-257.

Wilkins, D.A. 1978. The measurement of tolerance to edaphic factors by means of root growth, New Phytologist, 80: 623-633. 